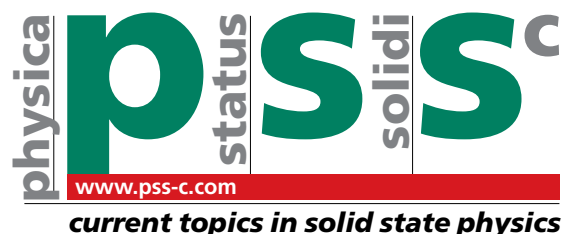

\title{
Sequential multiple-step europium ion implantation and annealing of $\mathrm{GaN}$
}

\author{
S. M. C. Miranda ${ }^{1,2}$, P. R. Edwards ${ }^{3}$, K. P. O'Donnell ${ }^{3}$, M. Boćkowski ${ }^{4}$, E. Alves ${ }^{1}$, I. S. Roqan ${ }^{5}$, \\ A. Vantomme ${ }^{2}$, and K. Lorenz ${ }^{*, 1}$ \\ ${ }^{1}$ Instituto Superior Técnico, Campus Tecnológico e Nuclear, Estrada Nacional 10, 2695-066 Bobadela LRS, Portugal \\ ${ }^{2}$ Instituut voor Kern- en Stralingsfysica, KU Leuven, 3001 Leuven, Belgium \\ ${ }^{3}$ SUPA Department of Physics, University of Strathclyde, Glasgow G4 0NG, Scotland, UK \\ ${ }^{4}$ Institute of High Pressure Physics, Polish Academy of Sciences, 01-142 Warsaw, Poland \\ ${ }^{5}$ King Abdullah University of Science and Technology (KAUST), Physical Science and Engineering Division, \\ Thuwal 23955-6900, Saudi Arabia
}

Received 14 June 2013, accepted 13 November 2013

Published online 16 January 2014

Keywords gallium nitride, ion implantation, crystal quality, europium

* Corresponding author: e-mail lorenz@ctn.ist.utl.pt, Phone: +351 21994 6000, Fax: +351 219946285

Sequential multiple Eu ion implantations at low fluence $\left(1 \times 10^{13} \mathrm{~cm}^{-2}\right.$ at $\left.300 \mathrm{keV}\right)$ and subsequent rapid thermal annealing (RTA) steps $\left(30 \mathrm{~s}\right.$ at $1000^{\circ} \mathrm{C}$ or $\left.1100^{\circ} \mathrm{C}\right)$ were performed on high quality nominally undoped $\mathrm{GaN}$ films grown by metal organic chemical vapour deposition (MOCVD) and medium quality GaN:Mg grown by hydride vapour phase epitaxy (HVPE). Compared to samples implanted in a single step, multiple implantation/annealing shows only marginal structural improvement for the MOCVD samples, but a significant improvement of crystal quality and optical activation of $\mathrm{Eu}$ was achieved in the HVPE films. This improvement is at- tributed to the lower crystalline quality of the starting material, which probably enhances the diffusion of defects and acts to facilitate the annealing of implantation damage and the effective incorporation of the Eu ions in the crystal structure. Optical activation of $\mathrm{Eu}^{3+}$ ions in the HVPE samples was further improved by high temperature and high pressure annealing (HTHP) up to $1400{ }^{\circ} \mathrm{C}$. After HTHP annealing the main room temperature cathodo- and photoluminescence line in $\mathrm{Mg}$-doped samples lies at $\sim 619 \mathrm{~nm}$, characteristic of a known Mg-related $\mathrm{Eu}^{3+}$ centre, while after RTA treatment the dominant line lies at $\sim 622 \mathrm{~nm}$, typical for undoped GaN:Eu.
1 Introduction The controlled introduction of rare earth (RE) ions into GaN presents an alternative route for optoelectronic devices with emission throughout the visible spectral range [1]. In particular, RE doping by ion implantation has been investigated in the past decade [2]. Ion implantation, used as a doping technique for nitrides, suffers less from solubility limits than in situ doping techniques and offers the possibility of controlled doping at precise depths below the surface and easy lateral pattering. However, it does cause considerable implantation damage [2-9]. Implantation of high fluences of heavy Eu ions leads to a reduced incorporation of Eu in substitutional Ga-sites and increases the density of non-radiative recombination centres $[8,9]$. In particular, it causes the formation of thermally stable extended defects such as stacking faults $[10$,
11]. It is therefore very important to minimize the damage caused during the implantation [7].

Although significant improvements of $\mathrm{Eu}^{3+}$ optical activation were reported recently using high annealing temperatures at high nitrogen pressure (HTHP), it was shown that, at temperatures even as high as $1450{ }^{\circ} \mathrm{C}$, full recovery of the crystal after implantation of high fluences could not be achieved [12-14]. On the other hand, we typically achieve complete recovery of the crystal structure by postimplant annealing at moderate temperature $\left(1000{ }^{\circ} \mathrm{C}\right)$ for low fluence implantation $\left(\sim 1 \times 10^{13} \mathrm{Eu} / \mathrm{cm}^{2}\right)[15,16]$.

In this study, we investigate the possibility of keeping the ion implantation damage in $\mathrm{GaN}$ low by performing multiple low fluence ion implantations. After each implantation step, annealing at moderate temperature is used to promote the structural recovery of the samples. 
2 Experimental details Commercial GaN wurtzite monocrystalline thin films, $\sim 3 \mu \mathrm{m}$ thick, grown on (0001) sapphire by metal organic chemical vapour deposition (MOCVD; Lumilog) were successively implanted with Eu to fluences of $1 \times 10^{13} \mathrm{Eu} / \mathrm{cm}^{2}$ (up to 10 times) resulting in a total implanted fluence of up to $1 \times 10^{14} \mathrm{Eu} / \mathrm{cm}^{2}$. Mgdoped GaN thin films grown by hydride vapour phase epitaxy (HVPE; TDI) were implanted up to 6 times with the same $1 \times 10^{13} \mathrm{Eu} / \mathrm{cm}^{2}$ fluence. The implantations were performed at $300 \mathrm{keV}$, room temperature (RT) and with the sample surface normal to the incident beam (channelled implantation) [7]. The samples were annealed after each implantation step in an ANNEALSYS rapid thermal annealing (RTA) furnace at $1000{ }^{\circ} \mathrm{C}$ for $30 \mathrm{~s}$ under flowing nitrogen. For comparison, selected samples were annealed after the final implantation step only or not annealed at all. For Mg-doped samples a final RTA step at $1100{ }^{\circ} \mathrm{C}$ was performed after the last implantation or implantation/annealing cycle.

In order to compare the damage caused by the implantations and the recovery achieved by annealing, high resolution X-ray diffraction (XRD) experiments were carried out using monochromated $\mathrm{Cu}-\mathrm{K}_{\alpha 1}$ radiation in a Bruker D8 Discover system equipped with a Göbel mirror, a $\mathrm{Ge}(220)$ double reflection asymmetric monochromator and a scintillation detector.

Cathodoluminescence $(\mathrm{CL})$ measurements were performed with a Cameca SX100 electron probe microanalyzer (EPMA) modified to perform RT CL, using a $10 \mathrm{keV}$ electron beam.

Additionally, 6 times implanted $\mathrm{GaN}: \mathrm{Mg}$ samples, with a total implanted fluence of $6 \times 10^{13} \mathrm{Eu} / \mathrm{cm}^{2}$, were annealed at $1100,1200,1300$ and $1400{ }^{\circ} \mathrm{C}$ for $30 \mathrm{~min}$ in $\mathrm{N}$ overpressure of $1 \mathrm{GPa}$ (HTHP annealing). RT photoluminescence (PL) measurements were performed on these samples using the $325 \mathrm{~nm}$ line of a $\mathrm{HeCd}$ laser.

3 Results and discussion Ion implantation in $\mathrm{GaN}$ epitaxial films introduces defects into the lattice which typically lead to high strain levels and to an expansion of the lattice parameter perpendicular to the surface [7, 17-19]. $\mathrm{XRD}$ is a sensitive technique to measure this strain and thus assess the damage induced during ion implantation and the subsequent recovery by annealing.

We measured XRD 2 $\theta-\omega$ (0002) curves for the asgrown, as-implanted and annealed GaN samples, as shown in Fig. 1, for the MOCVD samples. The results clearly show a pronounced secondary peak at lower angles than the main diffraction peak after implantation, revealing an expansion of the c-lattice parameter in the implanted region. The main diffraction peak arises from the undamaged film below the implanted region, while the shoulder is the result of strain induced into the implanted layer close to the surface. Figure 1a shows how the secondary peak in unannealed samples shifts to lower diffraction angles revealing first an increase of the strain with increasing fluence and then a saturation for the higher fluences in agreement with our previous work [17]. XRD curves after the first three cycles of implantation/annealing are shown in Fig. 1b. After annealing the secondary peak is almost completely removed for a single implantation step as well as for two implantation cycles. However, for samples with three and more implantation cycles, although the secondary peak shifts back towards higher angles, the expansion is not fully reversed. Figures $1 \mathrm{c}$ and $1 \mathrm{~d}$ present a comparison of the spectra corresponding to the final fluences of $5 \times 10^{13} \mathrm{Eu} / \mathrm{cm}^{2}$ and $1 \times 10^{14} \mathrm{Eu} / \mathrm{cm}^{2}$, respectively. It is clear that, for both fluences, only a marginal improvement is obtained by performing multiple annealing steps instead of just one RTA treatment. Figure 1d presents the curves of the sample implanted ten times, with $(10 \times \mathrm{i}-10 \times \mathrm{RTA})$ and without $(10 \times i-9 \times$ RTA $)$ the final RTA step, and also the samples implanted just once, annealed $(1 \times \mathrm{i}-1 \times \mathrm{RTA})$ or not $(1 \times i$-No RTA). The comparison between these samples reveals the higher strain states for the higher final implanted fluence, in particular after annealing.
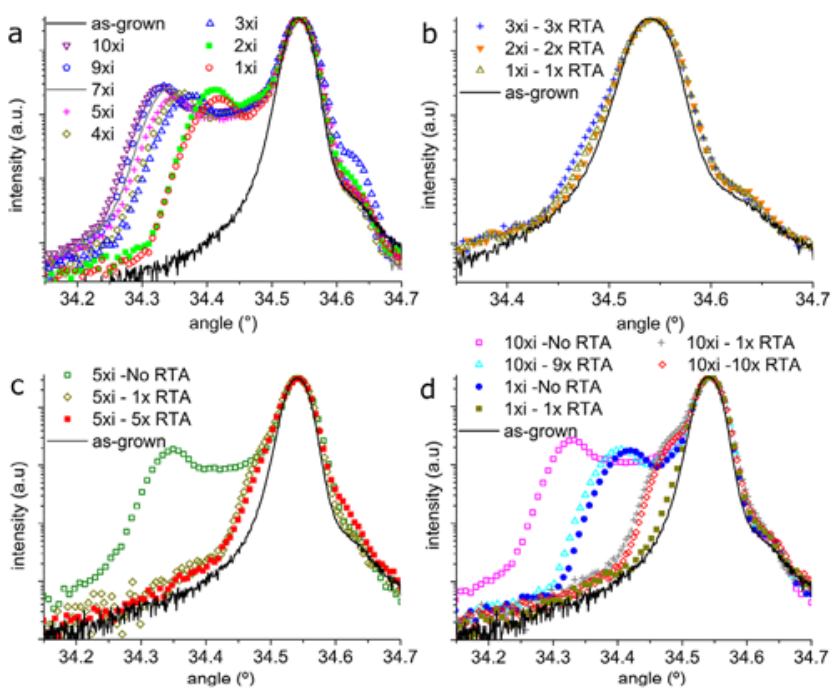

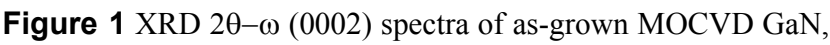
Eu implanted and annealed samples.

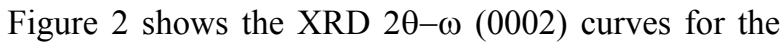
Mg-doped HVPE GaN samples. Surprisingly, the width of the main diffraction peak of the unimplanted material reduces significantly after annealing at $1000{ }^{\circ} \mathrm{C}$ (Fig. 2a). Broadening of the $2 \theta-\omega(0002)$ curves is mainly caused by a heterogeneous strain along the c-axis. In the present case, this heterogeneous strain may be related with native defects whose density can be reduced by annealing. This behaviour complicates the interpretation of the XRD data after implantation and annealing. Nevertheless, even for 6 successive implantation/annealing cycles, no pronounced shoulder remains at the lower angle side of the main diffraction peak indicating that the vast majority of the implantation damage is removed (Fig. 2a). Some improvement is seen for the sample with 6 annealing cycles compared to the sample with only one final annealing step at 
$1000{ }^{\circ} \mathrm{C}$ (Fig. 2b). Furthermore, Fig. 2c shows that annealing just once after 6 implantations, either at $1000{ }^{\circ} \mathrm{C}$ or $1100{ }^{\circ} \mathrm{C}$, yields the same strain state. In contrast, after running through 6 implantation/annealing cycles at $1000{ }^{\circ} \mathrm{C}$, one additional annealing step at $1100{ }^{\circ} \mathrm{C}$ promotes further structural recovery of the sample. Finally, Fig. $2 d$ compares the XRD curves for the two GaN materials revealing narrower curves and therefore lower heterogeneous strain for the MOCVD material even when compared with the annealed HVPE sample.
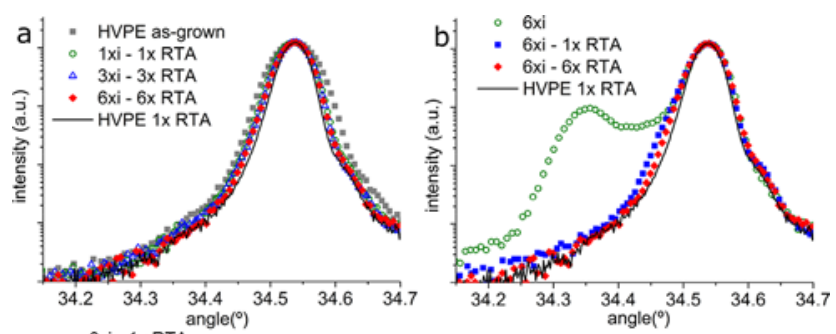

6xi - 1X RTA

6xi-1X RTA $1100^{\circ} \mathrm{C}$

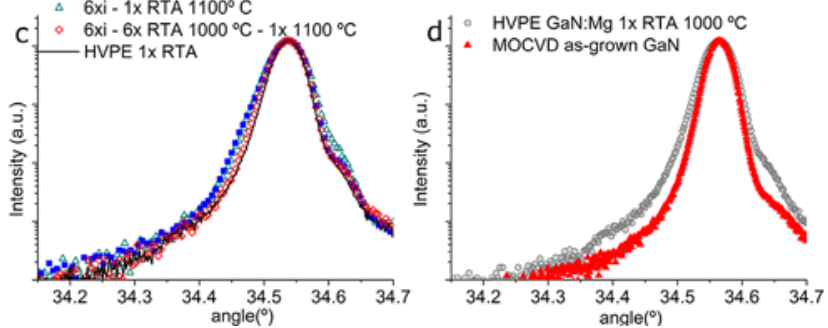

Figure $2(a-c)$ XRD 2 $\theta-\omega(0002)$ spectra of as-grown, Eu implanted and annealed HVPE GaN:Mg samples. d) Comparison of the unimplanted samples.

The above described results reveal better implantation damage recovery for the HVPE samples with the use of multiple implantation and annealing cycles; this indicates that, because of the lower crystalline quality of the asgrown HVPE samples, this strategy is beneficial to promote structural recovery during the annealing. Such behaviour can be understood in terms of point defect mobility. An increased number of native point defects and broken bonds may enhance the mobility of defects during annealing and result in a better recovery of low implantation damage. Note that this does not mean that the final result is a better crystal since the overall crystal quality may still be worse (however this is difficult to assess and quantify).

Optical activation of $\mathrm{Eu}^{3+}$ ions is achieved in all samples after annealing. Figure 3 a shows RT CL spectra for the MOCVD samples after several implantation/annealing cycles. The integrated CL intensity of the ${ }^{5} \mathrm{D}_{0} \rightarrow{ }^{7} \mathrm{~F}_{2}$ transitions of the $\mathrm{Eu}^{3+}$ ions around $622 \mathrm{~nm}$ is seen to increase linearly with the implantation fluence; however, with a proportionality factor below unity (when normalising intensity and fluence to the sample implanted with $1 \times 10^{13} \mathrm{Eu} / \mathrm{cm}^{2}$ a linear fit reveals a slope of $\sim 0.46$ ). Performing multiple implantation/annealing cycles, instead of only one annealing step after the final implantation, does not translate into significant improvement of the CL intensity (Fig. 3b).

The CL spectra after several implantation/annealing cycles for the Eu implanted GaN:Mg HVPE samples and annealing at $1000{ }^{\circ} \mathrm{C}$ are shown in Fig. 4a. The integrated CL intensity is shown in Fig. $4 \mathrm{~b}$ as a function of the final implantation fluence revealing again a linear behaviour. In fact, in these samples, we see a direct correlation between the total implanted fluence and the CL emission intensity showing a slope close to unity (when normalising intensity and fluence to the sample implanted with $1 \times 10^{13} \mathrm{Eu} / \mathrm{cm}^{2}$ a linear fit reveals a slope of $\sim 0.82$ ). For the $6 \times 10^{13} \mathrm{Eu} / \mathrm{cm}^{2} \mathrm{HVPE}$ implanted sample we measure an integrated CL intensity of $\sim 47500$ counts while for the MOCVD sample it reaches only $\sim 34500$ counts (see Fig. $3 b$ ) for the same implanted fluence. Since for a single implantation of $1 \times 10^{13} \mathrm{Eu} / \mathrm{cm}^{2}$ the intensities in the two materials are comparable, this improvement can be attributed to the reduction of defect concentration by sequential implantation/annealing, in good agreement with the structural results. In fact, for the HVPE samples a significant improvement is observed for sequential implantation/annealing cycles compared to the sample implanted to the highest fluence in just one step. We see that the multiple implantation and annealing strategy improves the CL emission intensity by effectively keeping the level of damage caused by the implantations low, while allowing a progressive build-up of the total implanted fluence.

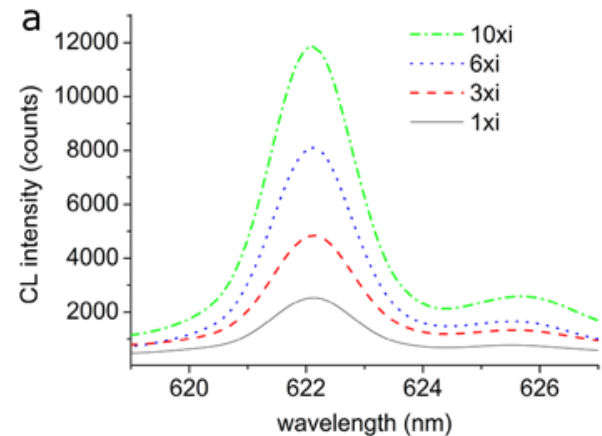

b

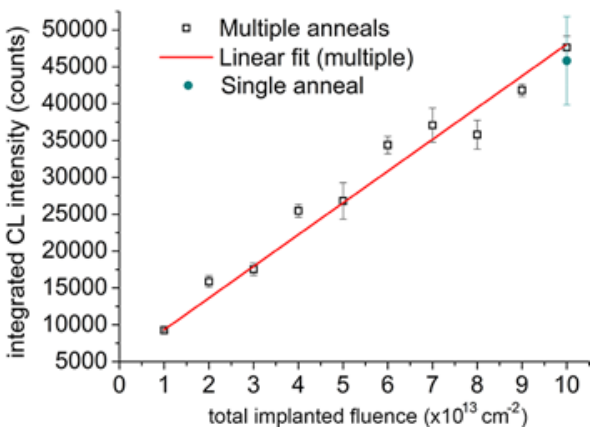

Figure 3 a) CL spectra of the MOCVD GaN, after different implantation/annealing cycles. b) Integrated CL intensity around the $622 \mathrm{~nm}$ peak after different implantation/annealing cycles as a function of the total implanted fluence. For comparison the intensity of a sample implanted to the maximum fluence and annealed just once is also shown. 
Figure $4 \mathrm{~b}$ shows that an increase in the final annealing temperature to $1100{ }^{\circ} \mathrm{C}$ actively contributes to further $\mathrm{Eu}$ activation in all samples in agreement with our previous reports [14]. To explore this improvement, the GaN:Mg samples, with a total implanted fluence of $6 \times 10^{13} \mathrm{Eu} / \mathrm{cm}^{2}$, were further annealed at $1100,1200,1300$ and $1400{ }^{\circ} \mathrm{C}$ in $\mathrm{N}$ overpressure of $1 \mathrm{GPa}$. The corresponding PL spectra are presented in Fig. 5a. Although we had achieved almost complete recovery of these samples already by RTA at $1100{ }^{\circ} \mathrm{C}$, we observe that annealing the samples at HTHP further promotes Eu activation. A linear trend of PL intensity increase with temperature is demonstrated in Fig. $5 b$ indicating that some of the residual defects are still being removed. The effective Eu activation requires high temperature annealing $[13,14]$ and the fact that the residual damage remains low throughout the implantations should lead to an optimized incorporation of $\mathrm{Eu}$ in substitutional Ga sites.
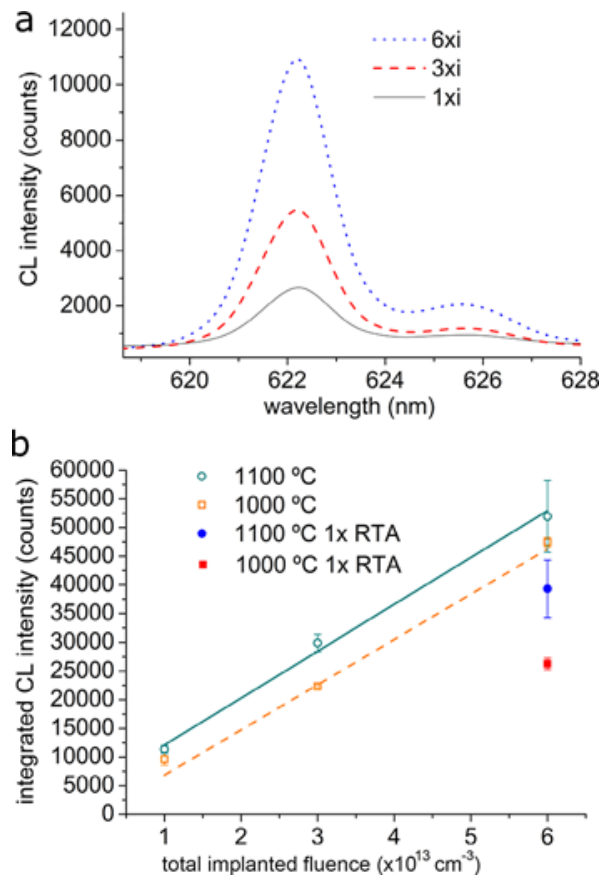

Figure 4 a) CL spectra of the HVPE GaN:Mg after different implantation/annealing cycles for RTA at $1000^{\circ} \mathrm{C}$. b) Integrated CL intensity around the $622 \mathrm{~nm}$ peak after different implantation/annealing cycles as a function of the total implanted fluence for samples annealed at $1000{ }^{\circ} \mathrm{C}$. The $\mathrm{CL}$ intensities for the same samples after a final RTA treatment at $1100{ }^{\circ} \mathrm{C}$ are also presented. For comparison the intensity of a sample implanted to the maximum fluence and annealed just once at either temperature is shown.

Another interesting effect of HTHP annealing is the formation of a new Mg-related Eu-centre, with the dominant emission line at $\sim 619 \mathrm{~nm}$ seen in both CL and PL spectra at RT. This centre has been recently reported in in situ $\mathrm{Eu} / \mathrm{Mg}$ co-doped MOCVD GaN [20] and an interest- ing temperature-hysteretic behaviour of this centre, named Eu0, in implanted samples was discussed in terms of a charge-transfer involving the shallow-deep instability of the $\mathrm{Mg}$ acceptor [21]. Our results show that the high nitrogen pressure and/or the longer annealing time play an important role in the formation of this Eu0 centre since it occurs, albeit very weakly, for HTHP annealing at $1100{ }^{\circ} \mathrm{C}$ while for low pressure RTA at the same temperature the known Eu1 and Eu2 centres [12,22] with main emission lines at $\sim 622 \mathrm{~nm}$, are dominant.
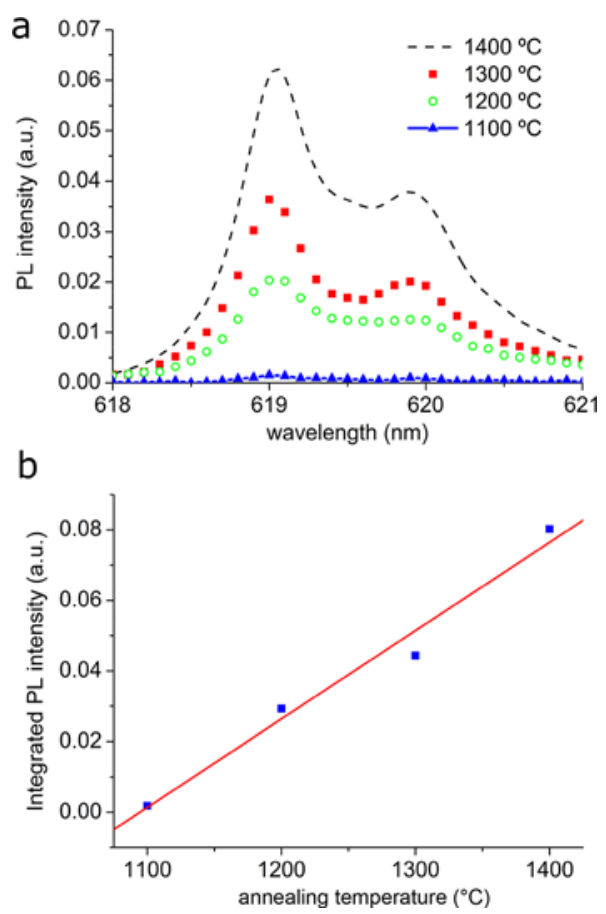

Figure 5 a) PL spectra of the Eu implanted GaN:Mg HTHP annealed samples. b) Integrated PL intensity as a function of the annealing temperature.

4 Conclusions Sequential multiple low fluence Eu ion implantations, each followed by an RTA step at $1000^{\circ} \mathrm{C}$ were performed on high quality MOCVD GaN and medium quality HVPE GaN:Mg samples.

The analysis of the XRD measurements evidences that residual damage remains in the Eu-implanted MOCVD GaN samples for multiple implantation/annealing cycles and recovery of the crystalline structure is only marginally improved compared to samples annealed only once after the last implantation. The measured CL intensity increases with increasing implantation fluence, but in agreement with the structural results, no significant difference is seen between the multiple annealed and the single annealed sample. No relevant improvement is achieved by using this method compared to the traditional one-time anneal procedure.

In contrast, a significant impact is obtained for the HVPE GaN:Mg samples with sequential implantation/annealing cycles compared to only one implantation 
and annealing procedure. We found that the damage can be significantly reduced in these samples, even in the multiple-implanted samples with total implanted fluence of $6 \times 10^{13} \mathrm{Eu} / \mathrm{cm}^{2}$. For these samples, the initial crystal quality was not so good allowing for better recovery. This potential for recovery was attributed to an increased defect mobility. We found the multiple implantation and annealing strategy to be effective in the GaN:Mg samples for keeping the damage level low and contributing to the enhancement of the CL emission intensity. HTHP annealing further increased the luminescent efficiency of these samples. For these annealing conditions a new Mg-related Eucentre, Eu0, becomes dominant with a main emission line at $\sim 619 \mathrm{~nm}$.

We conclude that the multiple implantation and annealing procedure may have potential for applications that do not require the highest crystal quality. Besides the discussed optical doping with RE ions this may be the case for devices relying on cheaper large area production, e.g., for solar cell applications.

Acknowledgements Financial support by FCT Portugal (PTDC/CTM/100756/2008, PTDC/CTM-NAN/2156/2012, PTDC/FIS-NAN/0973/2012, Investigador FCT) is gratefully acknowledged. This work has been supported by the European Community as the following Integrating Activities: Support of Public and Industrial Research Using Ion Beam Technology (SPIRIT) and Supporting Postgraduate Research with Internships in industry and Training Excellence (SPRITE).

\section{References}

[1] K. P. O'Donnell and V. Dierolf (eds.), Rare-Earth Doped III-Nitrides for Optoelectronic and Spintronic Applications (Spinger, Dordrecht, 2010).

[2] K. Lorenz, E. Alves, F. Gloux, and P. Ruterana, Top. Appl. Phys. 124, 25 (2010).

[3] S. O. Kucheyev, J. S. Williams, and S. J. Pearton, Mater. Sci. Eng. R 33, 51 (2001).

[4] E. Alves, K. Lorenz, R. Vianden, C. Boemare, M. J. Soares, and T. Monteiro, Mod. Phys. Lett. B 15, 1281 (2001).

[5] E. Wendler, A. Kamarou, E. Alves, K. Gärtner, and W. Wesch, Nucl. Instrum. Meth. Phys. Res. B 206, 1028 (2003).

[6] K. Lorenz, U. Wahl, E. Alves, T. Wojtowicz, P. Ruterana, S. Ruffenach, and O. Briot, Superlattices Microstruct. 36, 737 (2004).

[7] B. Pipeleers, S. M. Hogg, and A. Vantomme, J. Appl. Phys. 98, 123504 (2005).

[8] K. Lorenz, N. P. Barradas, E. Alves, I. S. Roqan, E. Nogales, R. W. Martin, K. P. O'Donnell, F. Gloux, and P. Ruterana, J. Phys. D: Appl. Phys. 42, 165103 (2009).

[9] A. Vantomme, B. De Vries, and U. Wahl, Top. Appl. Phys. 124, 55 (2010).

[10] F. Gloux, T. Wojtowicz, P. Ruterana, K. Lorenz, and E. Alves, J. Appl. Phys. 100, 073520 (2006).

[11] A. Turos, Radiat. Eff. Defects Solids 168, 431 (2013).

[12] I. S. Roqan, K. P. O’Donnell, R. W. Martin, P. R. Edwards, S. F. Song, A. Vantomme, K. Lorenz, E. Alves, and M. Boćkowski, Phys. Rev. B 81, 085209 (2010).
[13] K. Lorenz, E. Alves, I. S. Roqan, K. P. O’Donnell, A. Nishikawa, Y. Fujiwara, and M. Boćkowski, Appl. Phys. Lett. 97, 111911 (2010).

[14] K. Lorenz, S. M. C. Miranda, E. Alves, I. S. Roqan, K. P. O'Donnell, and M. Boćkowski, Proc. SPIE 8262, 82620C-1 (2012).

[15] S. M. C. Miranda, P. Kessler, J. G. Correia, R. Vianden, K. Johnston, E. Alves, and K. Lorenz, Phys. Status Solidi C 9, 1060 (2012).

[16] E. Alves, M. F. da Silva, J. G. Marques, J. C. Soares, and K. Freitag, Mater. Sci. Eng. B 59, 207 (1999).

[17] B. Lacroix, S. Leclerc, A. Declémy, K. Lorenz, E. Alves, and P. Ruterana, EPL 96, 46002 (2011).

[18] N. Catarino, E. Nogales, N. Franco, V. Darakchieva, S. M. C. Miranda, B. Méndez, E. Alves, J. G. Marques, and K. Lorenz, EPL 97, 68004 (2012).

[19] A. Vantomme, S. M. Hogg, M. F. Wu, B. Pipeleers, M. Swart, S. Goodman, D. Auret, K. Iakoubovskii, G. J. Adriaenssens, K. Jacobs, and I. Moerman, Nucl. Instrum. Methods B 175-177, 148 (2001).

[20] D. Lee, A. Nishikawa, Y. Terai, and Y. Fujiwara, Appl. Phys. Lett. 100, 171904 (2012).

[21] K. P. O'Donnell, R. W. Martin, P. R. Edwards, K. Lorenz, E. Alves, and M. Boćkowski, Temperature-dependent hysteresis of the emission spectrum of Eu-implanted, Mg-doped HVPE GaN, accepted in AIP Proceedings of ICPS (Zürich, Switzerland, 2012).

[22] L. Bodiou, A. Braud, J.-L. Doualan, R. Moncorgé, J. H. Park, C. Munasinghe, A. J. Steckl, K. Lorenz, E. Alves, and B. Daudin, J. Appl. Phys.105, 043104 (2009). 